\title{
Cognitive performance of healthy young rats following chronic donepezil administration
}

\author{
Debora Cutuli • Francesca Foti • Laura Mandolesi • \\ Paola De Bartolo • Francesca Gelfo • \\ Francesca Federico $\cdot$ Laura Petrosini
}

Received: 3 August 2007 / Accepted: 18 January 2008

(C) Springer-Verlag 2008

\begin{abstract}
Rationale Experimental studies have investigated the effects of chronic donepezil treatment on the behavioral deficits elicited by reduced activity or the loss of cholinergic neurons that occurs in aging or in models of dementia. However, few studies have analyzed the effects of chronic donepezil treatment on the cognitive functions of intact animals.

Objectives The cognitive functions of healthy young rats treated chronically with the acetylcholinesterase inhibitor donepezil were evaluated using a wide behavioral test battery. Results Chronic treatment with donepezil ameliorated memory functions and explorative strategies, speeded up the acquisition of localizing knowledge, augmented responsiveness to the context, and reduced anxiety levels. However, it did not affect spatial span, modify motivational levels, or influence associative learning.

Conclusions The present findings show the specific profile of donepezil action on cognitive functions in the presence of unaltered cholinergic neurotransmission systems.
\end{abstract}

D. Cutuli $\cdot$ F. Foti $\cdot$ L. Mandolesi $\cdot$ P. D. Bartolo $\cdot$ F. Gelfo •

F. Federico $\cdot$ L. Petrosini

IRCCS Santa Lucia Foundation,

Rome, Italy

L. Mandolesi $\cdot$ F. Gelfo

University of Naples "Parthenope",

Naples, Italy

D. Cutuli · F. Foti · P. D. Bartolo $\cdot$ L. Petrosini $(\bowtie)$

Department of Psychology, University of Rome "La Sapienza",

Via dei Marsi 78,

00185 Rome, Italy

e-mail: laura.petrosini@uniroma1.it

F. Federico

University of Siena,

Siena, Italy
Keywords Acetylcholinesterase inhibitor · Donepezil · Behavioral test battery $\cdot$ Mnesic functions .

Cognitive enhancer $\cdot$ Cholinergic neurotransmission

\section{Introduction}

Acetylcholine $(\mathrm{ACh})$ is a pivotal neurotransmitter in learning, memory, and attentive functions (Giovannini et al. 1997; Parent and Baxter 2004; Pepeu and Giovannini 2004). Alteration of cholinergic neurons is one of the primary pathological changes found in the brains of patients affected by Alzheimer's disease (AD; Birks and Flicker 2006). The "cholinergic hypothesis" (Bartus et al. 1982; Bartus 2000; Freo et al. 2002; Sarter et al. 2003; Phillis 2005), which postulates that the age-related decline in cognition may be correlated with reduced cerebral cholinergic function, has led to the development of pro-cholinergic compounds, such as acetylcholinesterase inhibitors (AChE-Is), which are able to enhance cognitive functioning (Dawson and Iversen 1993; Carey et al. 2001; Takada et al. 2003).

Donepezil is a second-generation AChE-I with fewer side effects than earlier drugs of its class and it has a very long half-life (about $72 \mathrm{~h}$ ). Currently, it is the first-line palliative treatment for improving cognitive functioning in AD patients afflicted by mild to moderate degrees of dementia (Rogers et al. 1998; Shigeta and Homma 2001; Sugimoto 2001; Bontempi et al. 2003; Seltzer 2007; Winstein et al. 2007). Furthermore, donepezil was reported to increase frontal activity during a working memory task in patients affected by mild cognitive impairment, and this increase was related to improved cognition (Saykin et al. 2004). There are also reports that donepezil reversed memory deficits in experimental models of learning and memory (Cheng et al. 1996; Rupniak et al. 1997; Higgins et 
al. 2002; Tokita et al. 2002; van der Staay and Bourger 2005; Wise et al. 2007). Acute administration of donepezil in experimental models of cholinergic deficits improved performances in the radial arm maze (Ogura et al. 2000; Wise et al. 2007), the Morris water maze (SpowartManning and van der Staay 2005), in spatial and visual recognition tasks (Kirkby et al. 1996; Poorheidari et al. 1998; Prickaerts et al. 2005), and in serial reaction time tasks (Kirkby et al. 1996).

Some experimental studies have investigated the effects of chronic donepezil treatment on the behavioral deficits elicited by reduced activity or the loss of cholinergic neurons that occurs in aging (Barnes et al. 2000; Hernandez et al. 2006) or in models of dementia (Dong et al. 2005; Yamada et al. 2005). In particular, in $\mathrm{Tg} 2576$ mice, an experimental model of $\mathrm{AD}$, donepezil had ameliorative effects on memory-related deficits (Dong et al. 2005). Furthermore, chronic donepezil administration improved memory impairment induced by i.c.v. injections of streptozotocin (Sonkusare et al. 2005). Thus, research has addressed the influence of donepezil on the symptoms provoked by altered cholinergic transmission. However, very few studies have investigated the putative influence of donepezil treatment on normal subjects. Acute donepeziltreated normal mice exhibited an increased rate of spontaneous alternations compared with controls (Spowart-Manning and van der Staay 2004). Moreover, acute donepezil administration before a spatial test elicited memory enhancing effects in rodents (Wise et al. 2007). These experimental data integrate a few human studies reporting the effects of cholinergic stimulation in healthy adult subjects. Davis et al. (1978) reported that acute physostigmine administration enhances storage of information in long-term memory as well as its retrieval, without alterations of short-term memory. More recently, it has been demonstrated that modulation of the cholinergic system by physostigmine infusions improved working memory efficiency and reduced activation of the cortical regions associated with working memory (Furey et al. 2000). Furthermore, Sun et al. (1999) reported improved memory and learning functions in adolescent Chinese students following a 4-week administration of an AChE-I (huperzine-A). Recent studies in healthy aircraft pilots reported beneficial effects on the retention of complex tasks (flight simulator) after 30 days of donepezil treatment (Yesavage et al. 2002; Mumenthaler et al. 2003). However, the tasks were very complex and tapped a combination of cognitive functions that were difficult to distinguish. Moreover, Grön et al. (2005) reported the selective enhancement of episodic memory performance in healthy young subjects following 30 days of donepezil treatment, suggesting that the hippocampal region is the major target of cholinergic enhancement elicited by longterm inhibition of AChE. Overall, these findings suggest that
AChE-Is, and donepezil in particular, may act as "cognition enhancers" in normal subjects.

Based on these findings, we set out to assess the specific effects of chronic donepezil treatment on unaltered cholinergic neurotransmission systems. To this aim, healthy young rats chronically treated with donepezil were evaluated using a behavioral test battery that tapped different cognitive functions.

\section{Materials and methods}

Animals

Twenty male Wistar rats $(300-350 \mathrm{~g})$ kept in standard laboratory conditions (08:00-20:00 light, food and water ad libitum) were used in the present experiments. The animals were maintained according to the guidelines for ethical conduct developed by the European Communities Council Directive of 24 November 1986 (86/609/EEC) and approved by the Ethical Committee on animal experiments of the University of Rome "La Sapienza". Rats were randomly assigned to two experimental groups: the treated group (D; $n=10)$ received daily i.p. injections of donepezil and the control group $(\mathrm{C} ; n=10)$ daily injections of saline.

\section{Drug}

For 3 weeks prior to the behavioral testing, donepezil (Eisai Inc.) was administered daily at a dosage of $0.5 \mathrm{mg} / \mathrm{kg}$; then, for the next 7 weeks it was administered daily at a dosage of $0.2 \mathrm{mg} / \mathrm{kg}$ dissolved in $0.5 \mathrm{ml}$ of $0.9 \% \mathrm{NaCl}$ solution. The same volume of saline, but without the drug, was administered daily to the control animals. All injections were administered at the end of the testing sessions to avoid any acute drug effects. According to clinical (Rogers et al. 1998; Rogers and Friedhoff 1996) and experimental (Wise et al. 2007; Ogura et al., 2000; Dong et al. 2005) studies, a $0.2 \mathrm{mg} / \mathrm{kg}$ dose of donepezil is sufficient to influence repeated behavioral testing but not to elicit heavy side effects. The initial dose of $0.5 \mathrm{mg} / \mathrm{kg}$ was chosen to habituate the rats to a high drug dosage that would be reduced during the behavioral testing phase.

\section{Behavioral testing}

As shown in Fig. 1, a battery of six tests was administered in the following order: Morris water maze (MWM), to analyze competence in building a spatial cognitive map and in using navigational strategies; radial arm maze (RAM), to analyze spatial reference and working memory; serial learning task (SLT), to analyze cognitive flexibility; open field (OF), to evaluate the ability to develop spatial and 


\section{FLOW DIAGRAM}

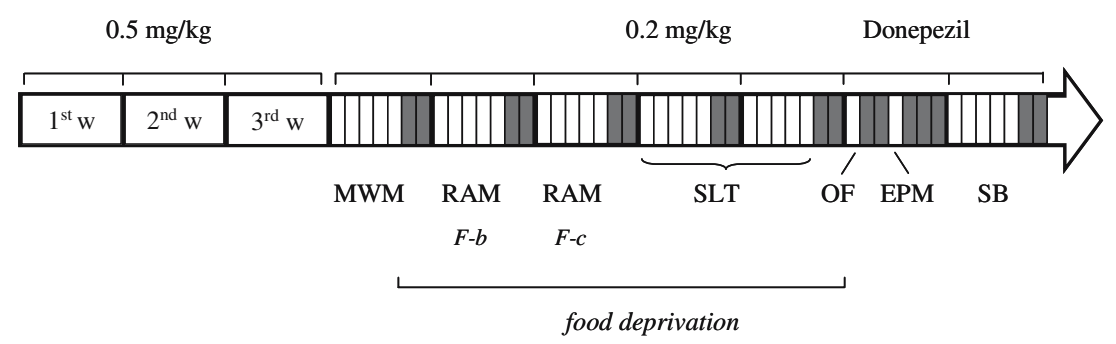

Fig. 1 Flow diagram of experimental procedures. After the first 3 weeks of $0.5 \mathrm{mg} / \mathrm{kg}$ donepezil administration, a battery of six tests, namely, $M W M$ Morris water maze, $R A M$ radial arm maze ( $F-b$ fullbaited and $F$-c forced-choice procedures), $S L T$ serial learning task, $O F$

discriminative competence; elevated plus maze (EPM), to assess anxiety levels; shuttle box (SB), to analyze active avoidance learning.

\section{Morris water maze (MWM)}

The rats were placed in a circular white pool (diameter $140 \mathrm{~cm}$ ) filled with $24^{\circ} \mathrm{C}$ water $(60 \mathrm{~cm}$ deep), made opaque by the addition of 21 of milk. An escape platform (diameter $10 \mathrm{~cm}$ ), submerged $2 \mathrm{~cm}$ below or elevated $2 \mathrm{~cm}$ above the water level, was placed in the middle of one cardinal quadrant, $30 \mathrm{~cm}$ from the pool walls. The rat was released into the water from randomly varied starting points and was allowed to swim around for $120 \mathrm{~s}$ to find the platform. Each rat underwent two sessions of four trials per day, with a 4-h inter-session interval. When the rat reached the platform, it was allowed to remain there for $30 \mathrm{~s}$. In the first four sessions, the platform was hidden in the northwest quadrant (place I); in the next two sessions, the platform was visible in the northeast quadrant (cue phase); in the final four sessions, the platform was hidden in the northeast quadrant (place II; Morris et al. 1982; Petrosini et al. 1996; Federico et al. 2006; Leggio et al. 2006). This protocol allowed investigating numerous components of spatial function. Place I analyzed the sequence of navigational strategies (spatial procedural learning) put into action to explore the pool and to find the platform, as well as the ability to build a spatial map (spatial memory) by using extra-maze cues to locate the hidden platform. The cue phase analyzed the development of stimulus-response (platform/reaching) associative learning and employment of the procedural knowledge acquired during place I. Finally, place II analyzed the ability to remodel the spatial map by exploiting the intra-maze information acquired in the cue phase in the presence of already acquired procedural strategies and also provided information about the plastic properties of spatial learning processes.

The rats' trajectories in the pool were monitored by a video camera mounted on the ceiling. Video signals were open field, $E P M$ elevated plus maze, $S B$ shuttle box, was performed with concomitant $0.2 \mathrm{mg} / \mathrm{kg}$ donepezil administration. All rats were food deprived during RAM and SLT tests. Gray boxes indicate testfree days

relayed to a monitor and to an image analyzer (Ethovision, Noldus, Wageningen, the Netherlands).

The following behavioral parameters were considered in analyzing the MWM performances: latencies to find the platform, total distance swum in the entire pool, distance traveled in a $20-\mathrm{cm}$ peripheral annulus, heading angles (the angle formed by the direction of the rat's head when leaving the pool edge and a straight line from the starting location to the platform), and swimming velocity.

\section{Radial arm maze (RAM)}

The apparatus consisted of a central platform (diameter $30 \mathrm{~cm})$ from which eight arms $(12.5 \mathrm{~cm}$ wide $\times 60 \mathrm{~cm}$ long $)$ radiated like the spokes of a wheel. A food well $(5 \mathrm{~cm}$ deep) was located at the end of each arm (Mandolesi et al. 2001). Prior to the habituation phase, the rats were food restricted to decrease their weight by $20 \%$.

Full-baited maze procedure In each session, all maze arms were baited with a piece of Purina chow. The rat was placed on the central platform and allowed to make eight correct visits, 16 (correct or incorrect) visits, or to explore the maze for $15 \mathrm{~min}$. The animals were submitted to two sessions a day for 5 consecutive days. The inter-session interval was $4 \mathrm{~h}$.

The following parameters were considered: total entries (number of visits, either correct or incorrect), total errors (number of re-visited arms), spatial span (longest sequence of correctly visited arms), and percentage of $45^{\circ}$ angles ( $45^{\circ}$ angles made in each session divided by the total number of angles made $\times 100$ ).

Forced-choice procedure Forty-eight hours after the end of the above-described protocol, all animals were submitted to the forced-choice paradigm. In the first phase, only four arms (for example, arms 1, 3, 4, and 7) were opened and baited and the remaining arms were closed. The baited arms were separated by different angles to prevent the animal from solving the problem by adopting a stereotyped pattern. 
The rat was allowed to explore the open arms. Then, it was put in its cage for $60 \mathrm{~s}$ before being returned to the maze. In the second phase, the rat was allowed free access to all eight arms, but only the four previously closed arms were baited. This task was repeated for 5 consecutive days with a different configuration of arms closed each day to avoid a fixed search pattern.

The parameters considered were working memory errors, considered as re-entries into already visited arms. In the second phase, this parameter was broken down further into two error subtypes: across-phase errors, defined as entries into an arm entered in the first phase; withinphase errors, defined as re-entries into an arm visited earlier in the same session.

\section{Serial learning task (SLT)}

The apparatus consisted of a white rectangular wooden box $(150 \times 40 \times 40 \mathrm{~cm})$ subdivided into five compartments (30 $\mathrm{cm}$ long) by four gray panels with two unidirectional doors (height $10 \mathrm{~cm}$, width $8 \mathrm{~cm}$ ). Each door could be locked using a pivot; therefore, if the animal pushed the door it opened about $2 \mathrm{~cm}$. The small split allowed the rat to introduce its muzzle but prevented it from going through the door. This trick allowed us to obtain proof that the animals were attempting to open the "incorrect" door. The entire apparatus was closed with a transparent Plexiglas cover. The final, rewarded compartment was darkened using a black cover.

The rats continued to be food restricted. Following a 3-day pre-training session, they were submitted to one testing session a day for 10 consecutive days. All daily sessions included 12 trials. In each trial, the goal was to reach the fifth compartment and collect the reward by going through the open doors and making no attempt to force open the closed ones. Each animal was given a sequence of open doors that remained unchanged for all 12 trials of a session but that changed every session; thus, each animal was tested in ten different sequences.

In each of the 12 trials of a session, the following parameters were analyzed: errors, i.e., the attempt to force open the closed door (in each trial this parameter ranged from 4 to 0 ); correct choices, i.e., the longest sequence of correct choices (in each trial this parameter ranged from 0 to 4); perseverations, i.e., the number of errors made at the same door in the 12 trials of a session. As the first session gave the animal time to become accustomed to the actual testing procedure, only the results of $\mathrm{S} 2-\mathrm{S} 9$ were considered.

\section{Open field (OF)}

The apparatus consisted of a circular container (diameter $140 \mathrm{~cm}$ ) delimited by a $30-\mathrm{cm}$-high wall. Five objects were present simultaneously in the open field: (1) a metal bar with a conical base, (2) a plunger, (3) a long steel rod, (4) a yellow rubber plug, and (5) a black cylinder with a plastic cup turned upside down on top of it.

During session 1 (S1), each rat was allowed to move freely in the empty open field and its baseline level of activity was measured. During S2-S4 (habituation phase), four objects were placed in a square arrangement in the middle annulus of the arena and the fifth one was placed in the central area. For S5 (spatial change, S5 and S6), the spatial configuration was changed by moving objects 2 and 5 so that the initial square arrangement was changed to a polygon-shaped configuration, without any central object. During S7 (novelty), the configuration was modified by substituting object 4 with a green plastic object shaped like a half moon. Sessions lasted $6 \mathrm{~min}$; inter-session intervals were $3 \mathrm{~min}$.

All testing was recorded by a video camera whose signal was relayed to a monitor and to the previously described image analyzer.

The parameters taken into account were total distance (in meters) traveled in the arena, distances traveled in the peripheral or central arena sectors, and time spent contacting objects (contact was considered to have taken place when the rat's snout actually touched an object or when it sniffed the object for at least $1 \mathrm{~s}$ ).

\section{Elevated plus maze (EPM)}

The maze, which was raised $90 \mathrm{~cm}$ above the ground was formed by a wooden structure in the shape of a cross with four $50 \mathrm{~cm} \times 10 \mathrm{~cm}$ arms. The north and south arms were open, but the east and west arms were enclosed by walls $36 \mathrm{~cm}$ high.

The following behavioral parameters were measured: number of defecation boluses, total time spent in the open and closed arms or on the platform, and frequency of entries into the arms.

\section{Shuttle box (SB)}

An active avoidance shuttle box (Ugo Basile type 7532, Comerio-Varese, Italy) with an electrifiable grid floor (steel rods spaced $1.5 \mathrm{~cm}$ apart) was used (Molinari et al. 1997). The area inside the box was divided into two compartments by a ceiling-to-floor partition that had a $10-\mathrm{cm}$ central opening to allow the animals to cross from one side to the other. Each compartment had a $15-\mathrm{W}$ bulb mounted on the ceiling. Each daily session consisted of 100 automatic computerized deliveries of conditioned (light, $15 \mathrm{~W}, 10 \mathrm{~s}$ ) and unconditioned (foot shock, $0.2 \mathrm{~mA}, 10 \mathrm{~s}$ ) stimuli. Sessions were repeated for 5 consecutive days.

The following parameters were considered: frequency and latency of avoidances (shuttle behavior occurring 
during light time), frequency and latency of escapes (shuttle behavior occurring when the foot shock was on), frequency of failures (remaining in the start compartment until the shock was turned off), and inter-trial crossings (repeated passing from one compartment to the other during the trial regardless of the light or the foot shock).

\section{Statistical analysis}

Metric unit results were compared by one-, two-, or threeway analyses of variance (ANOVAs) followed by post hoc multiple comparisons using Duncan's test. Differences were considered significant at $p<0.05$.

\section{Results}

Morris water maze

As the sessions went by, all animals displayed a progressive reduction of latencies in reaching the platform (Fig. 2a). A two-way ANOVA (treatment $\times$ session) on latency values revealed significant treatment $(F(1,18)=4.1 ; p<0.05)$ and session $(F(9,162)=35.1 ; p<0.00001)$ effects. Interaction was also significant $(F(9,162)=2.14 ; p<0.02)$. As indicated by post hoc comparisons, at the start of the MWM task there were no significantly different latency values between the two groups of animals. However, in the next two place I sessions the treated animals displayed lower latencies than the controls. Interestingly, a significant difference between groups was also found in the first session of the cue phase, where the treated animals reached the visible platform more quickly than the controls. A two-way ANOVA (treatment $\times$ session) on the total distance swum in the pool to reach the platform revealed no significant treatment effect $(F(1,18)=$ $0.01 ; p$ n.s.), but the session effect was highly significant $(F(9,162)=27.33 ; p<0.00001$; Fig. 2b). Interaction was also significant $(F(9,162)=3.24 ; p<0.001)$. The mean velocity of the treated animals was $27.14 \mathrm{~cm} / \mathrm{s}$, and that of the controls $22.7 \mathrm{~cm} / \mathrm{s}$ (one-way ANOVA: $F(1,18)=$ $15.07 ; p<0.001)$. A two-way ANOVA (treatment $\times$ session) on velocity values revealed significant treatment $(F(1,18)=$ $14.99 ; p<0.001)$ and session $(F(9,162)=3.38 ; p<0.001)$ effects. Interaction was also significant $(F(9,162)=2.41$; $p<0$. 01). Post hoc comparisons revealed significant increases in velocity values during the 2 nd and 3 rd sessions of place I. Thus, the latency reduction seemed to be related to differences in velocity rather than to total distances. Interesting results were found when the distance traveled in the peripheral annulus was analyzed. Although all animals tended to reduce their percentage of peripheral swimming progressively $(F(9,162)=5.28 ; p<0.00001)$ as the sessions went by, the treated animals swam in the peripheral sectors significantly less than the controls $(F(1,18)=46.5 ; p<$ $0.00001)$ throughout the task. Interaction was not significant $(F(9,162)=1.38 ; p$ n.s.; Fig. $2 c)$.

To obtain information about the localizatory knowledge on platform position gained by the animals as the sessions went by, the heading angles were calculated. Once again, the two experimental groups exhibited the same heading angles at the beginning of the task; however, from the end of place I the treated animals displayed more pointed localizing behavior, which was maintained throughout the task (Fig. 2d). A two-way ANOVA revealed significant treatment $(F(1,18)=6.41 ; p<0.02)$ and session $(F(9,162)=$ $9.35 ; p<0.00001)$ effects. Interaction was not significant $(F(9,162)=1.75 ; p$ n.s. $)$.

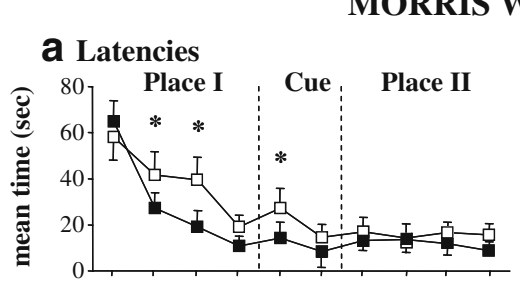

\section{MORRIS WATER MAZE}
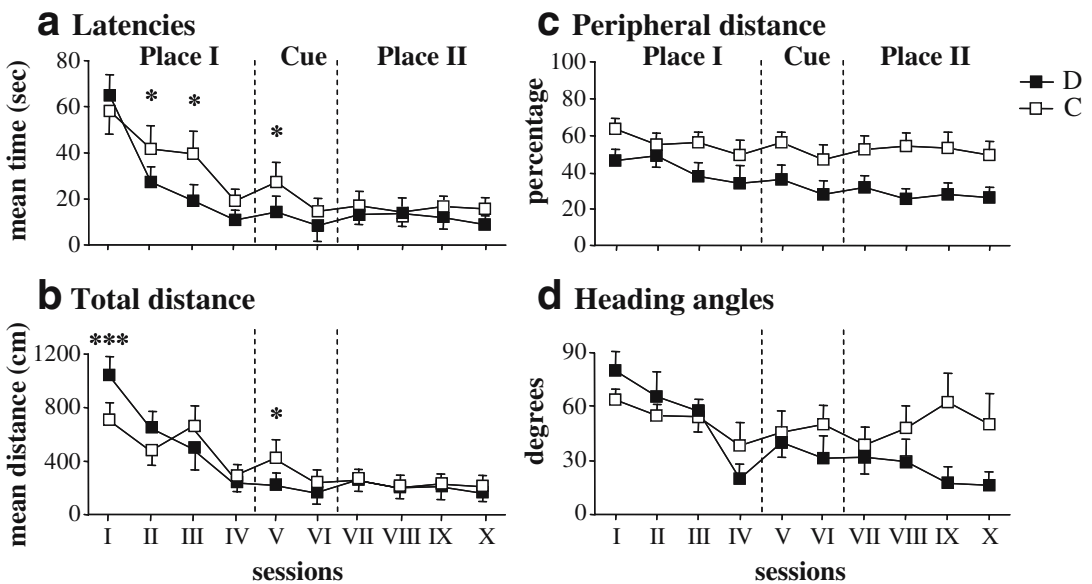

d Heading angles

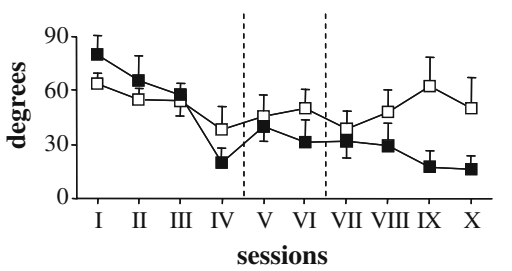

Fig. 2 Effects of chronic donepezil treatment on the MWM performances of healthy young rats. Mean escape latencies to reach the platform (a), total distance swum in the whole arena (b), percentage of distance swum in a $20-\mathrm{cm}$ peripheral annulus (c), and heading angles

(d) displayed by the two experimental groups are depicted. In this and in the following figures, $D$ donepezil-treated animals, $C$ control animals. Asterisks indicate post hoc comparisons (Duncan's test) between groups. ${ }^{*} p<0.05,{ }^{* * *} p<0.001$. Vertical bars indicate SEM 
Radial arm maze

\section{Full-baited procedure}

As the sessions went by, all animals decreased their entries and errors significantly (entries: $F(9,162)=6.18 ; p<0.0001$; errors: $F(9,162)=7.18 ; p<0.0001)$; however, the treated animals made significantly fewer entries and errors than the controls (entries: $F(1,18)=6.41 ; p<0.02$; errors: $F(1,18)=$ $7.12 ; p<0.02)$. Neither interaction was significant (entries: $F(9,162)=1.4$; $p$ n.s.; errors: $F(9,162)=1.5$; $p$ n.s.; Fig. 3a). No effect of treatment on the spatial span was found, as revealed by a two-way ANOVA (treatment: $F(1,18)=0.05$; p n.s.; session: $F(9,162)=4.15 ; p<0.0001$; interaction: $F$ $(9,162)=0.47 ; p$ n.s. $)$.

\section{RADIAL ARM MAZE}

\section{Full-baited procedure}
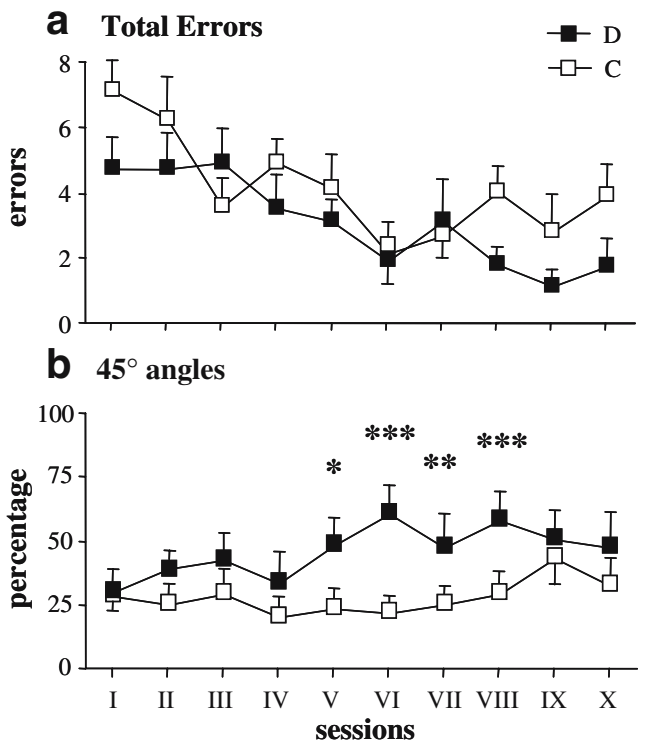

Forced-choice procedure

C Second Phase Errors

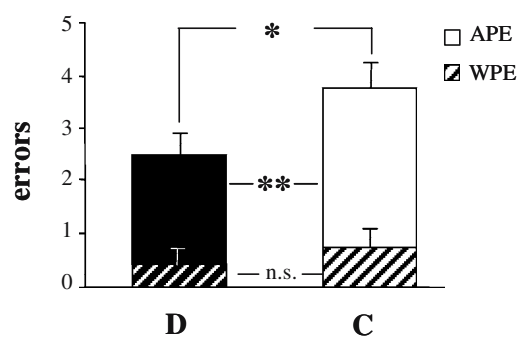

Fig. 3 Effects of chronic donepezil treatment on the RAM performances of healthy young rats. Total errors (a) and $45^{\circ}$ angles (b) displayed by the two experimental groups in the full-baited procedure are depicted. In c, errors displayed by the two experimental groups in the forced-choice procedure are illustrated. Asterisks indicate post hoc comparisons (Duncan's test) between groups. ${ }^{*} p<0.05,{ }^{* *} p<0.01$, $* * * p<0.001$. Vertical bars indicate SEM. APE Across-phase errors, WPE within-phase errors
As illustrated in Fig. 3b, the percentages of $45^{\circ}$ angles followed different time courses in the two experimental groups. Although their starting values were similar to those of the controls, the treated animals made higher percentages of $45^{\circ}$ angles from the 5 th session on and, in the last two sessions, displayed the same $45^{\circ}$ angle percentages as the controls. A two-way ANOVA revealed significant treatment $(F(1,18)=10.68 ; p<0.04)$ and session $(F(9,162)=2.97 ; p<$ $0.01)$ effects. Interaction was also significant $(F(9,162)=$ $2.14 ; p<0.03)$. A correlation analysis between $45^{\circ}$ angles and errors of the two experimental groups in the ten RAM sessions was performed (Table 1). In treated animals, Pearson's $r$ evolved from very low positive values to high negative values. This pattern was different from that of the controls whose Pearson's $r$ values were low and negative in the first and last sessions and positive in the middle sessions. These findings show a different use of mnesic and procedural competences in the two experimental groups.

\section{Forced-choice procedure}

The treated group, which already performed better than the controls in the first phase (two-way ANOVA: treatment: $F$ $(1,18)=10.69, p<0.01$; sessions: $F(4,72)=8.22, p<0.001$; interaction: $F(4,72)=4.12, p<0.01)$, made significantly fewer errors even in the second phase (two-way ANOVA: treatment: $F(1,18)=4.34, p=0.05$; sessions: $F(4,72)=4.85$, $p<0.01$; interaction: $F(4,72)=3.90, p<0.01$; Fig. $3 c)$.

An additional error analysis revealed that treated animals made fewer across-phase errors than controls (two-way ANOVA: treatment: $F(1,18)=6.71 ; p<0.02$; session: $F$ $(4,72)=3.82 ; p<0.01$; interaction: $F(4,72)=4.98 ; p<$ $0.001)$. However, no difference was found between groups for within-phase errors (Fig. 3c).

Serial learning task

A significant difference in the total number of errors made by the two groups was revealed by one-way ANOVA ( $F$ $(1,18)=7.79 ; p<0.01$; Fig. 4a). The treated group showed fewer perseverations than the controls (one-way ANOVA: $F(1,18)=4.33 ; p<0.05$; Fig. $4 \mathrm{~b})$. The longest sequences of correct choices, which were made in the 2nd and the 10th session, were compared by means of a two-way ANOVA (treatment $\times$ session). This analysis revealed significant treatment $(F(1,18)=21.4 ; p<0.001)$ and session $(F(1,18)=$ $145.59 ; p<0.00001)$ effects. Interaction was also significant $(F(1,18)=22.03 ; p<0.001)$. In both groups, post hoc comparisons indicated a significant lengthening of the sequence of correct choices from the 2 nd to the 10th sessions $(p<0.0001)$; however, the treated animals performed better in both sessions (Fig. 4c). 
Table 1 Correlation between $45^{\circ}$ angles and errors made by the experimental groups during RAM full-baited procedure

\begin{tabular}{|c|c|c|c|c|c|c|c|c|c|c|}
\hline \multirow[t]{2}{*}{ Group } & \multicolumn{10}{|c|}{ Session } \\
\hline & I & II & III & IV & $\mathrm{V}$ & VI & VII & VIII & IX & $X$ \\
\hline $\mathrm{D}$ & 0.06 & 0.36 & -0.11 & -0.05 & -0.14 & -0.73 & -0.62 & -0.56 & -0.61 & -0.32 \\
\hline $\mathrm{C}$ & -0.45 & -0.55 & -0.49 & 0.39 & 0.26 & 0.47 & 0.13 & -0.16 & -0.24 & -0.51 \\
\hline
\end{tabular}

$D$ Donepezil-treated group, $C$ control group

The errors made by both experimental groups in the 12 trials of the 2nd, 5th, and 10th sessions were compared by means of a three-way ANOVA (treatment $\times$ session $\times$ trial), which revealed significant treatment $(F(1,18)=13.61 ; p<$

\section{SERIAL LEARNING TASK}

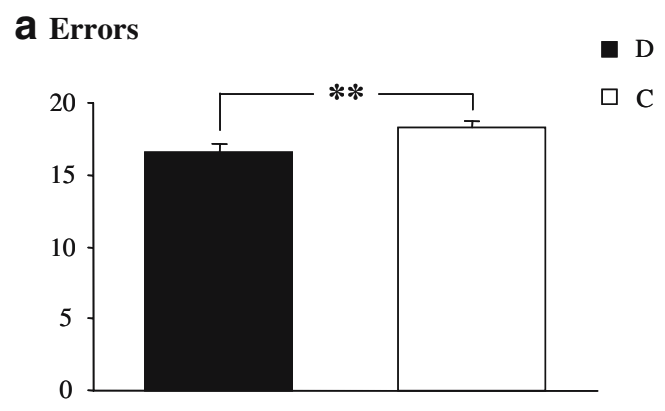

b Perseverations

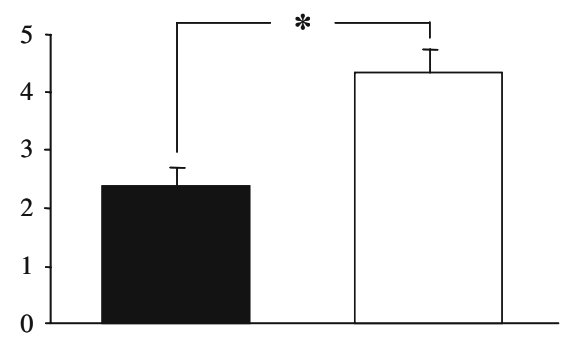

C Sequence of correct choices

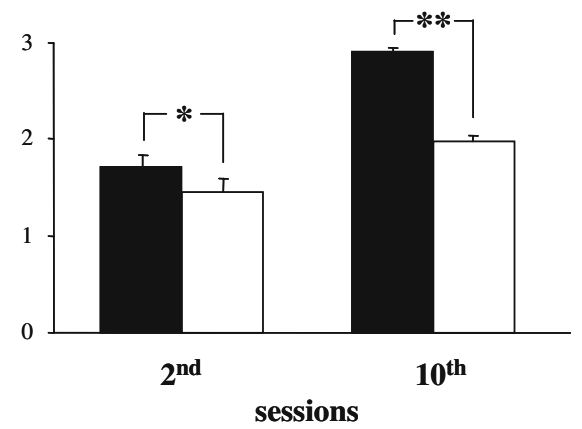

Fig. 4 Effects of chronic donepezil treatment on the SLT performances of healthy young rats. Mean errors (a), perseverations (b), and the longest sequences of correct choices displayed by the two experimental groups are depicted. Asterisks indicate post hoc comparisons (Duncan's test) between groups. ${ }^{*} p<0.05,{ }^{* *} p<0.01$. Vertical bars indicate SEM
$0.001)$, session $(F(2,36)=59.20 ; p<0.00001)$ and trial $(F$ $(11,198)=22.57 ; p<0.00001)$ effects. The interaction treatment $\times$ trial was significant $(F(11,198)=1.95 ; p<0.03)$, but the interaction treatment $\times$ session was not $(F(2,36)=0.79$; $p$ n.s.). The among-factor interaction was also significant $(F$ $(22,396)=1.71 ; p<0.02)$. Thus, the treated animals improved their performances and progressively reduced their errors more than the controls within the same session (the performances of the 12th trial were typically better than those of the first trial) and throughout the task (the performances of the 10th session were better than those of the first session; Fig. 5).

\section{SERIAL LEARNING TASK}

Errors within trials and sessions
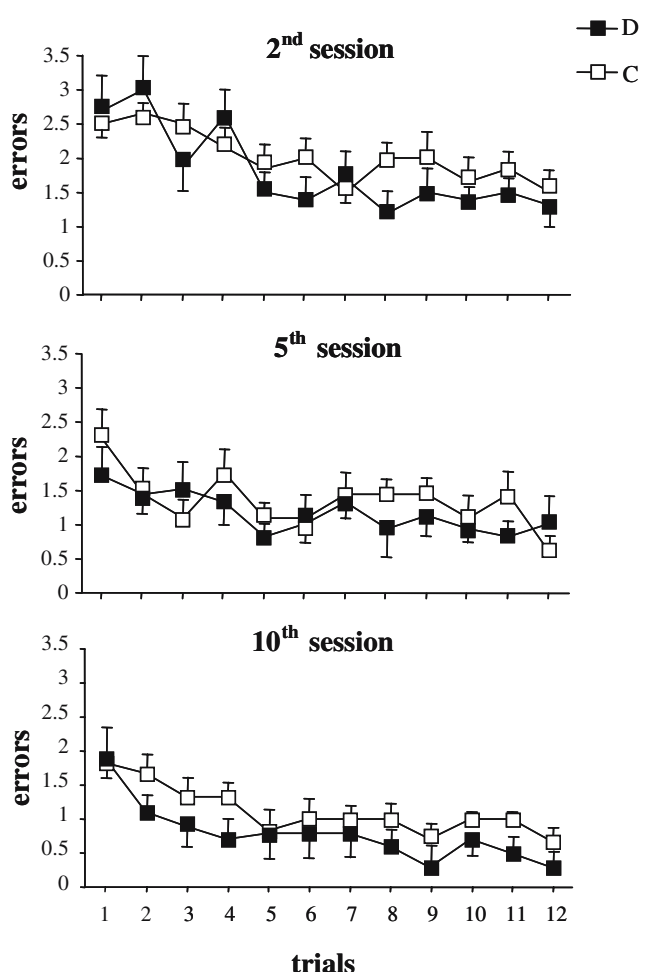

Fig. 5 Effects of chronic donepezil treatment on the SLT performances of healthy young rats. Errors made by the two experimental groups in the 12 trials of the 2nd, 5th, and 10th sessions are depicted. Vertical bars indicate SEM 
Open field

The absence of significant differences in the number of defecation boluses $(F(1,18)=0.29 ; p$ n.s. $)$ indicated that all animals exhibited a comparable level of anxiety. Only the session effect was significant $(F(6,108)=17.20 ; p<$ 0.00001 ), given the already scanty number of boluses collapsed as the task went by. Further, motionless time during the task did not significantly differ between the two groups $(F(1,18)=1.02 ; p$ n.s. $)$. Only the session effect was significant, because of the prolonged time spent motionless, as the sessions went by $(F(6,108)=14.41 ; p<0.00001)$. No significant differences in number of rearings were observed (two-way ANOVA: treatment: $F(1,18)=1.52 ; p$ n.s.; session: $F(6,108)=10.27 ; p<0.00001$; interaction: $F(6,108)=$ $0.44 ; p$ n.s.). Regarding the distance traveled within the arena during the seven sessions, a two-way ANOVA revealed that, while the treatment effect $(F(1,18)=1.50$; $p$ n.s.) was not significant, the session effect $(F(6,108)=$ 20.04; $p<0.00001)$ and the interaction $(F(6,108)=2.45 ; p<$ 0.03 ) were significant (Fig. 6a). As revealed by post hoc comparisons on distances traveled in the single sessions by the two groups, in S1, S2, and S7, the treated animals traveled longer distances than the controls. To evaluate which sectors (peripheral or central) of the arena were explored most, one-way ANOVAs on distances traveled in $\mathrm{S} 1, \mathrm{~S} 2$, and $\mathrm{S} 7$ revealed that the central sectors (containing the objects in $\mathrm{S} 2$ and $\mathrm{S} 7$ ) were significantly more explored by the treated animals in $\mathrm{S} 2(F(1,18)=4.43 ; p<0.05)$ and $\mathrm{S} 7(F$ $(1,18)=4.18 ; p<0.05)$. In $\mathrm{S} 1$, no difference between groups $(F(1,18)=1.3 ; p$ n.s. $)$ in traveling the central sectors was found (treated group: $\bar{x}: 20.23 \pm 2.71 ; \bar{x}: 17.49 \pm 5.39$ ).

When objects were present, all animals showed habituation (Fig. 6b). However, the habituation features were influenced by the treatment $(F(1,18)=5.14 ; p<0.04)$, because in $\mathrm{S} 2$ the treated animals tended to maintain contact with the objects longer than the controls. Session effect $(F(2,36)=39.00 ; p<0.001)$ and interaction were also significant $(F(2,36)=7.53 ; p<0.01)$.

As revealed by a three-way ANOVA (treatment $\times$ session $\times$ object), during the spatial change (S5 and S6) all rats explored the displaced objects more than the non-

\section{OPEN FIELD TEST}

\section{a Total distance travelled}

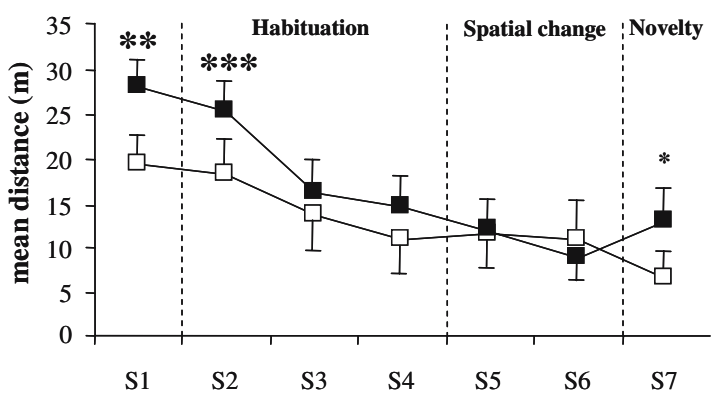

b Habituation phase

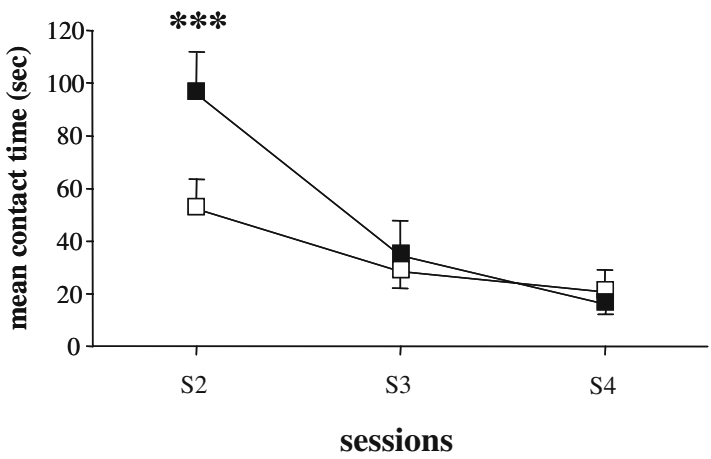

Fig. 6 Effects of chronic donepezil treatment on the OF performances of healthy young rats. Total distance traveled in the arena throughout the seven sessions of the task (a), mean contact time with objects during habituation phase (S2-S4; b), spatial change (S5-S6; c), and novelty (S7; d) of the two experimental groups are depicted. Asterisks
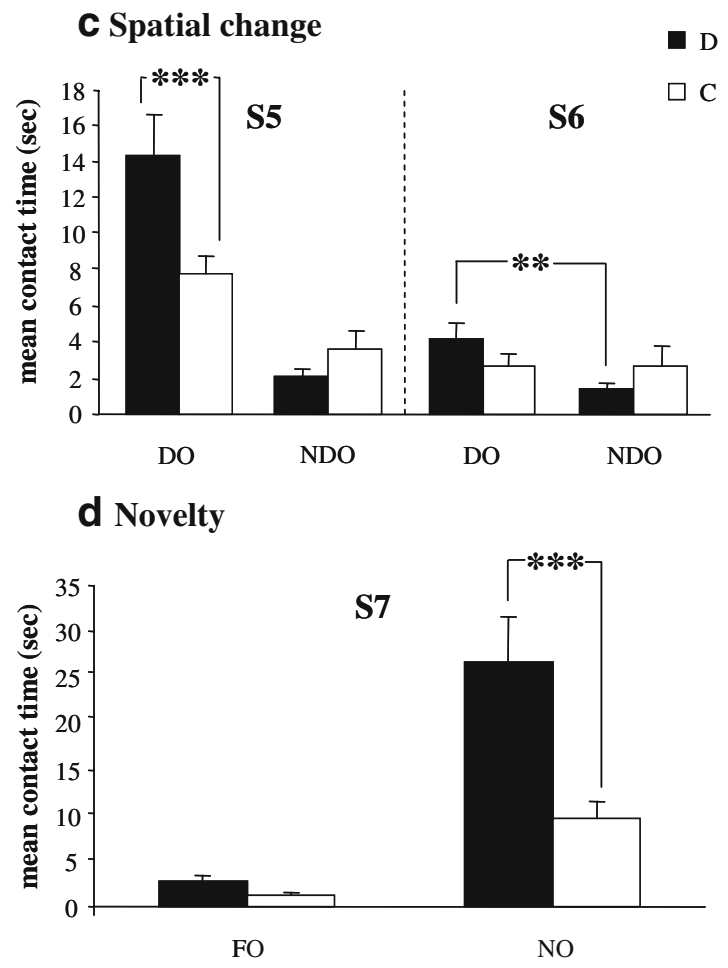

indicate post hoc comparisons (Duncan's test) between groups. ${ }^{*} p<$ $0.05,{ }^{* *} p<0.01, * * * p<0.001$. Vertical bars indicate SEM. In $C, N D O$ non-displaced objects, $D O$ displaced objects. In $D, F O$ familiar objects, $N O$ novel object 
displaced ones $(F(1,18)=54.1 ; p<0.0001)$; thus, the treatment effect on the spatial change was not significant $(F$ $(1,12)=1.63 ; p$ n.s.). Post hoc comparisons performed on the significant second-order interaction $(F(1,18)=5.69 ; p<$ 0.03 ) provided interesting results. In $\mathrm{S} 5$, the treated animals contacted the displaced objects significantly $(p<0.0002)$ longer than the controls and they continued to display significantly $(p<0.004)$ longer contact with the displaced objects in S6. Note that this "strategy" of durable context exploration by the treated animals was not shared by the controls, which showed no difference in contact times between displaced and not-displaced objects in S6 (Fig. 6c).

During novelty, in both groups of animals the contact time with the familiar objects was very brief, but the treated animals contacted the novel object longer than the controls. A two-way ANOVA (treatment $\times$ object) revealed significant treatment $(F(1,18)=10.38 ; p<0.005)$ and object $(F$ $(1,18)=37.93 ; p<0.00001)$ effects. Interaction was also significant $(F(1,18)=8.65 ; p<0.01$; Fig. $6 \mathrm{~d})$.

\section{Elevated plus maze}

While the controls spent more time in the closed arms, the treated animals, which did not exhibit the normal open arm avoidance, spent a similar amount of time in both kinds of arms, as illustrated in Fig. 7. In fact, when the differences in the time spent in the closed vs. open arms by the two experimental groups were analyzed by means of a one-way ANOVA, a significant treatment effect was found $(F(1,18)=$ $4.52 ; p<0.05)$. Once more, no significant difference in defecation boluses was found between groups. To verify the presence of hyperactive behaviors, the frequencies of entries in the arms were analyzed. As no significant treatment effect was evident in the frequency of entries into the arms $(F(1,18)=0.10 ; p$ n.s. $)$, hyperactive effects of donepezil can be excluded.

\section{ELEVATED PLUS MAZE}

Time

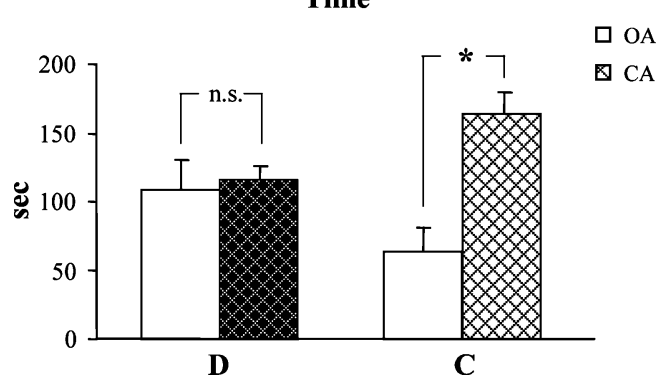

Fig. 7 Effects of chronic donepezil treatment on the EPM performances of healthy young rats. Time spent in open arms $(O A)$ and closed arms $(C A)$ by the two experimental groups is depicted. The asterisk indicates the post hoc comparison (Duncan's test) between groups. ${ }^{*} p<0.05$. Vertical bars indicate SEM
Shuttle box

Both groups exhibited an almost complete absence of escape failures $(\mathrm{D}: \bar{x}=4.4 \pm 2.7 ; \mathrm{C}: \bar{x}=6.2 \pm 3.8)$ and inter-trial crossings $(\mathrm{D}: \bar{x}=1.1 \pm 0.4 ; \mathrm{C}: \bar{x}=2.2 \pm 0.7)$. The progressive decrease in escape frequencies was associated with a parallel increase in avoidances during the five testing sessions. Two-way ANOVAs (treatment $x$ session) failed to reveal significant treatment effects in escape and avoidance frequencies, while the session effect was highly significant for both responses (escapes: treatment $F(1,18)=0.29 ; p$ n.s.; sessions $F(4,72)=23.68 ; p<$ 0.00001 ; interaction $F(4,72)=0.3 ; p$ n.s.; avoidances: treatment $F(1,18)=0.67 ; p$ n.s.; sessions $F(4,72)=20.31$; $p<0.00001$; interaction $F(4,72)=1.17 ; p$ n.s.). The same statistical pattern was found when the latencies of avoidances and escapes were examined. This latter result seems to be linked to intrinsic features of the active avoidance task, in which simple conditioning (light/shock) was required. In fact, both groups of animals obtained good learning performances.

\section{Discussion}

Few studies in healthy humans have addressed changes in cognitive function provoked by chronic administration of AChE-Is in the presence of unaltered cholinergic transmission (Ernst et al., 2001; Yesavage et al. 2002; Kumari et al. 2003; Gron et al. 2005), and there are even fewer studies in animals (Barnes et al., 2000; Dong et al. 2005; Hernandez et al. 2006). However, it seems important to assess the cognitive performances of healthy subjects treated chronically with AChE-Is. In fact, even in healthy subjects it is important to analyze the difference between long-term administration and acute single-bolus injection of AChEIs, because it is crucial that a drug which is effective in the acute form also be effective with repeated administrations. Furthermore, manipulation of cholinergic neurotransmission in subjects in which cholinergic functioning is not altered by pharmacological agents or physiological aging may shed light on the effects of the so-called cognitive enhancers, or, as Rose (2002) termed them, the "smart drugs".

To verify whether specific cognitive functions (and in the affirmative case, which ones) were influenced by sustained cholinergic enhancement in healthy rats, we used a large battery of tests that tapped very different functions including spatial or non-spatial, associative or discriminative, mnesic or attentive, and procedural or mapping competences.

Cholinergic enhancement positively influenced functions that require a significant working memory load; it speeded 
up the acquisition of procedural competences, ameliorated functions linked to declarative (localizatory) memory, augmented responsiveness to the context, and reduced anxiety levels. Conversely, the treatment did not affect short-term spatial memory storage and did not modify motivational levels or influence associative learning. Specifically, at the start of the MWM task the treated animals exhibited the same latencies as the controls but quickly acquired significantly lower latencies, suggesting improved mnesic and mapping abilities (hidden platform) as well as increased attention to contextual cues (visible platform; Petrosini et al. 1996; Federico et al. 2006; Leggio et al. 2006). Moreover, they exhibited reduced peripheral swimming throughout the testing. In general, the treated animals showed improved spatial learning and increased motor activity, as indicated by their higher swimming velocity. Furthermore, they acquired more pointed (although not necessarily more efficient) navigational strategies, as indicated by their narrow heading angles. The present findings are in agreement with Hernandez et al. (2006) who reported that aged rats chronically treated with donepezil displayed superior performances in reaching the hidden MWM platform. Further, it has been found that the spatial deficits induced by bilateral lesions of entorhinal cortex are partially antagonized by acute donepezil treatment (SpowartManning and van der Staay 2005).

In the full-baited RAM procedure, the treated group made significantly fewer errors than the control group. Successful performance on the RAM task requires working memory and mapping abilities as well as efficient procedural competences. In fact, to avoid repeated entries in already visited arms the subjects have to remember the arm just visited, represent the maze in a cognitive spatial map, and/or use efficient procedural strategies. A general evaluation of the behavior of both groups indicates that in the first sessions the controls reduced their errors by using (even if only partially) procedural competences, while in the middle sessions they mainly employed working memory abilities. Conversely, in the middle sessions the treated rats primarily exploited procedural competences and reduced working memory load, as indicated by their progressively correlated use of efficient strategies $\left(45^{\circ}\right.$ angles) to diminish errors (Table 1). Given their high $45^{\circ}$ angle percentage, it was necessary to distinguish procedural from working memory requirements, which the forcedchoice procedure allowed. In this procedure, the treated animals' errors were significantly reduced. Thus, it can be suggested that, depending on the context, the treated animals were able to reorganize their strategies and shift from using spatial procedures to applying mnesic competences. The present RAM data fit with the improved working memory performances displayed by normal mice treated acutely with donepezil and tested in a T-maze continuous alternation task (Spowart-Manning and van der Staay 2004). Accordingly, in normal rats acute donepezil treatment reduced errors in a delay RAM procedure (Wise et al. 2007). Evidently, the beneficial effects of acute donepezil treatment on working memory competences were maintained in the case of chronic treatment.

In the OF task, cholinergic treatment increased explorative tendencies, as indicated by the longer distances traveled by the treated animals in S1, S2, and S7. Note that the treated animals foraged the arena and, especially, visited the middle area containing the objects. Overall, these findings suggest that the treated animals were more responsive to the context. In fact, they were more active when they faced a new context (in S1, a new empty open field) or when it dramatically changed (in S2, an open field with five new objects; in S7, the introduction of an unknown object). The treated animals' greater responsiveness to the context was revealed by their prolonged contact times with the displaced or novel objects in S5-S6 and S7. Once again, this result is in line with acute donepezil effects in spatial and visual memory tasks in the presence of scopolamine-induced cholinergic hypofunction (Kirkby et al. 1996; Rupniak et al. 1997; Poorheidari et al. 1998). Accordingly, in intact rats acute donepezil administration improved object recognition memory (Prickaerts et al. 2005).

The cholinergic treatment does not seem to elicit hyperactive tendencies. In fact, in OF the treated animals spent their time contacting the objects and did not forage around the arena (Fig. 6); in RAM they displayed a low number of entries; in EPM they exhibited the same entry frequencies as the controls; in SB they did not show augmented activity, as indicated by inter-trial crossings.

Interestingly, in EPM the treated animals spent the same amount of time in the closed arms as in the open ones, indicating they had low levels of anxiety. This finding fits with the benefits of donepezil in treating the behavioral symptoms of dementia (Levy et al. 1999; Gauthier et al. 2002a, b; Palmer 2002). As anxiety levels can affect learning and memory functions, it cannot be excluded that a component linked to the decreased anxiety exhibited by the treated animals could have influenced (even if only partially) their performances.

Overall, the present results show that treated animals exhibit accelerated acquisition and enhancement of both working and long-term memory together with rapid transition from one competence to another (for example, from procedural to mnesic competence). These better performances could be linked to a more complex mnesic representation, with stronger connections among cues, and to quick learning of procedural competences, driving explorative strategies more confidently. Overall, the treated animals seemed to process information more efficiently and 
to employ adaptive strategies more quickly than the controls. In other words, cognitive flexibility-a process as fundamental as memory in information processingappeared to be another factor favorably influenced by cholinergic treatment. In fact, it was to be expected that the treated animals would also show improved performances on tasks such as serial learning and, indeed, this was the case. In fact, in SLT treated animals demonstrated increasing efficiency in learning the repeated changes in the same problem. They displayed fewer errors than the controls and progressively reduced their errors both within the same session and throughout the sessions. Accordingly, the treated animals exhibited longer sequences of correct choices and reduced perseverations compared with the controls. While the daily task of detecting and remembering the correct sequence of choices primarily tapped mnesic functions, the daily task of facing different and unpredictable sequences of choices primarily tapped flexibility behaviors. The present data indicate that cholinergic enhancement made it possible for the animals to learn either the correct sequence of choices or how to efficiently switch among changing response rules.

The possibility that the improvements displayed by the treated animals were due to changes in motivation rather than to specific effects on learning and memory processes was considered. However, the cholinergic manipulation did not appear to affect motivational components by modifying the salience of the reward/punishment. In fact, all of the animals reached and promptly climbed onto the MWM platform; they always consumed the food pellets in RAM and SLT, and showed comparable response latencies (avoidances and escapes) in SB.

In conclusion, the present data highlight the positive effects of chronic donepezil treatment on mnesic abilities as well as on procedural, discriminative, and adaptive functions. There is some disagreement about how the cholinergic system promotes memory performances. According to some authors, mnesic improvement is driven directly by ACh (Gold 2003), while according to others it is indirectly mediated by enhancement of attentional functions (Everitt and Robbins 1997; Gill et al. 2000). Still others argue that $\mathrm{ACh}$ affects interactions between mnesic and attentive functions (Sarter et al. 2003; Parent and Baxter 2004). Specifically, cholinergic activation is considered a prerequisite of sustained attention (Sarter et al. 2001) which, in turn, appears to be a prerequisite of information acquisition, recall, and appropriate responses to environmental stimuli (Pepeu and Giovannini 2004). This approach was supported by neuroimaging experiments on volunteers subjected to a visual working memory task. It showed that physostigmineinduced cholinergic enhancement improved memory performance by augmenting the selectivity of perceptual processing during encoding (Furey et al. 2000). Following this line, it is not surprising that the treated animals exhibited enhanced mnesic capacities, as shown by MWM and RAM results, and superior discriminative abilities, as shown by OF results, although the two components may be difficult to disentangle.

It has been suggested that mechanisms other than enhancement of cholinergic functioning may also be responsible for donepezil efficacy in improving cognitive functions (Narahashi et al. 2004). In addition to its effects on $\mathrm{ACh}$, donepezil is reported to increase levels of norepinephrine, dopamine, and serotonin (Giacobini et al 1996; Zhang et al. 2004; Hatip-al-Khatib et al. 2005; Liang and Tang 2006; Shearman et al 2006), and to augment the activity of the NMDA system (Moriguchi et al 2005). However, as donepezil interactions with non-cholinergic neurotrasmettitorial systems have only been investigated in a few studies, using heterogeneous methodologies, different drug dosages, and evaluating cortical or subcortical regions, it is difficult to draw any final conclusions. On the other hand, the cholinergic projections are so widely diffused (Levin and Simon 1998; Pepeu and Giovannini 2004) that an interaction with other neurotransmitter systems is quite feasible.

Taken together, the present findings reveal the faceted action of donepezil on cognitive functions in the presence of unaltered cholinergic neurotransmission, suggesting that AChE-Is may be beneficial even in the case of specific cholinergic hypofunctions, targeting some symptoms more than others. This specific action of donepezil on cognitive function may be an expression of the not yet fully understood role of $\mathrm{ACh}$ in cognition.

Acknowledgments Donepezil was generously gifted by Eisai, Inc. This study was supported by MIUR grants to LP. None of the authors has a conflict of interest.

Conflict of interest statement None of the authors has any conflict of interest.

\section{References}

Barnes CA, Meltzer J, Houston F, Orr G, McGann K, Wenk GL (2000) Chronic treatment of old rats with donepezil or galantamine: effects on memory, hippocampal plasticity and nicotinic receptors. Neuroscience 99:17-23

Bartus RT (2000) On neurodegenerative diseases, models, and treatment strategies: lessons learned and lessons forgotten a generation following the cholinergic hypothesis. Exp Neurol 163:495-529

Bartus RT, Dean RL, Beer B, Lippa AS (1982) The cholinergic hypothesis of geriatric memory dysfunction. Science 30:408-414

Birks J, Flicker L (2006) Donepezil for mild cognitive impairment. Cochrane Database Syst Rev 193:CD006104

Bontempi B, Whelan KT, Risbrough VB, Lloyd GK, Menzaghi F (2003) Cognitive enhancing properties and tolerability of cholinergic agents in mice: a comparative study of nicotine, 
donepezil, and SIB-1553A, a subtype-selective ligand for nicotinic acetylcholine receptors. Neuropsychopharmacology 28:1235-1246

Carey GJ, Billard W, Binch H 3rd, Cohen-Williams M, Crosby G, Grzelak M, Guzik H, Kozlowski JA, Lowe DB, Pond AJ, Tedesco RP, Watkins RW, Coffin VL (2001) SCH 57790, a selective muscarinic $\mathrm{M}(2)$ receptor antagonist, releases acetylcholine and produces cognitive enhancement in laboratory animals. Eur J Pharmacol 431:189-200

Cheng DH, Ren H, Tang XC (1996) Huperzine a novel promising acetylcholinesterase inhibitor. Neuroreport 8:97-101

Davis KL, Mohs RC, Tinklenberg JR, Pfefferbaum A, Hollister LE, Kopell BS (1978) Physostigmine: improvement of long-term memory processes in normal humans. Science 201:272-274

Dawson GR, Iversen SD (1993) The effects of novel cholinesterase inhibitors and selective muscarinic receptor agonists in tests of reference and working memory. Behav Brain Res 57:143-153

Dong H, Csernansky CA, Martin MV, Bertchume A, Vallera D, Csernansky JG (2005) Acetylcholinesterase inhibitors ameliorate behavioral deficits in the $\mathrm{Tg} 2576$ mouse model of Alzheimer's disease. Psychopharmacology 181:145-152

Ernst M, Matochik JA, Heishman SJ, Van Horn JD, Jons PH, Henningfield JE, London ED (2001) Effect of nicotine on brain activation during performance of a working memory task. Proc Natl Acad Sci U S A 98:4728-4733

Everitt BJ, Robbins TW (1997) Central cholinergic systems and cognition. Annu Rev Psychol 48:649-684

Federico F, Leggio MG, Neri P, Mandolesi L, Petrosini L (2006) NMDA receptor activity in learning spatial procedural strategies II. The influence of cerebellar lesions. Brain Res Bull 16:356-367

Freo U, Pizzolato G, Dam M, Ori C, Battistin L (2002) A short review of cognitive and functional neuroimaging studies of cholinergic drugs: implications for therapeutic potentials. J Neural Transm 109:857-870

Furey ML, Pietrini P, Haxby JV (2000) Cholinergic enhancement and increased selectivity of perceptual processing during working memory. Science 290:2315-2319

Gauthier S, Feldman H, Hecker J, Vellas B, Ames D, Subbiah P, Whalen E, Emir B, Donepezil MSAD Study Investigators Group (2002a) Efficacy of donepezil on behavioral symptoms in patients with moderate to severe Alzheimer's disease. Int Psychogeriatr 14:389-404

Gauthier S, Feldman H, Hecker J, Vellas B, Emir B, Subbiah P, Donepezil MSAD Study Investigators' Group (2002b) Functional, cognitive and behavioral effects of donepezil in patients with moderate Alzheimer's disease. Curr Med Res Opin 18:347-354

Giacobini E, Zhu XD, Williams E, Sherman KA (1996) The effect of the selective reversible acetylcholinesterase inhibitor E2020 on extracellular acetylcholine and biogenic amine levels in rat cortex. Neuropharmacology 35:205-211

Gill TM, Sarter M, Givens B (2000) Sustained visual attention performance-associated prefrontal neuronal activity: evidence for cholinergic modulation. J Neurosci 20:4745-4757

Giovannini MG, Casamenti F, Bartolini L, Pepeu G (1997) The brain cholinergic system as a target of cognition enhancers. Behav Brain Res 83:1-5

Gold PE (2003) Acetylcholine modulation of neural systems involved in learning and memory. Neurobiol Learn Mem 80:194-210

Grön G, Kirstein M, Thielscher A, Riepe MW, Spitzer M (2005) Cholinergic enhancement of episodic memory in healthy young adults. Psychopharmacology 182:170-179

Hatip-Al-Khatib I, Iwasaki K, Yoshimitsu Y, Arai T, Egashira N, Mishima K, Ikeda T, Fujiwara M (2005) Effect of oral administration of zanapezil (TAK-147) for 21 days on acetylcholine and monoamines levels in the ventral hippocampus of freely moving rats. Br J Pharmacol 145:1035-44
Hernandez CM, Gearhart DA, Parikh V, Hohnadel EJ, Davis LW, Middlemore ML, Warsi SP, Waller JL, Terry AV (2006) Comparison of galantamine and donepezil for effects on nerve growth factor, cholinergic markers, and memory performance in aged rats. J Pharmacol Exp Ther 316:679-694

Higgins GA, Enderlin M, Fimbel R, Haman M, Grottick AJ, Soriano M, Richards JG, Kemp JA, Gill R (2002) Donepezil reverses a mnemonic deficit produced by scopolamine but not by perforant path lesion or transient cerebral ischaemia. Eur J Neurosci 15:1827-1840

Kirkby DL, Jones DN, Barnes JC, Higgins GA (1996) Effects of anticholinesterase drugs tacrine and E2020, the 5-HT(3) antagonist ondansetron, and the $\mathrm{H}(3)$ antagonist thioperamide, in models of cognition and cholinergic function. Behav Pharmacol 17:513-525

Kumari V, Gray JA, ffytche DH, Mitterschiffthaler MT, Das M, Zachariah E, Vythelingum GN, Williams SC, Simmons A, Sharma T (2003) Cognitive effects of nicotine in humans: an fMRI study. Neuroimage 19:1002-1013

Leggio MG, Federico F, Neri P, Graziano A, Mandolesi L, Petrosini L (2006) NMDA receptor activity in learning spatial procedural strategies. I. The influence of hippocampal lesions. Brain Res Bull 16:347-355

Levin ED, Simon BB (1998) Nicotinic acetylcholine involvement in cognitive function in animals. Psychopharmacology 138:217-30

Levy ML, Cummings JL, Kahn-Rose R (1999) Neuropsychiatric symptoms and cholinergic therapy for Alzheimer's disease. Gerontology 45:15-22

Liang YQ, Tang XC (2006) Comparative studies of huperzine A, donepezil, and rivastigmine on brain acetylcholine, dopamine, norepinephrine, and 5-hydroxytryptamine levels in freely-moving rats. Acta Pharmacol Sin 27:1127-36

Mandolesi L, Leggio MG, Graziano A, Neri P, Petrosini L (2001) Cerebellar contribution to spatial event processing: involvement in procedural and working memory components. Eur J Neurosci 14:2011-2022

Molinari M, Grammaldo LG, Petrosini L (1997) Cerebellar contribution to spatial event processing: right/left discrimination abilities in rats. Eur J Neurosci 9:1986-1992

Moriguchi S, Zhao X, Marszalec W, Yeh JZ, Narahashi T (2005) Modulation of $N$-methyl-D-aspartate receptors by donepezil in rat cortical neurons. J Pharmacol Exp Ther 315:125-135

Morris RG, Garrud P, Rawlins JN, O’Keefe J (1982) Place navigation impaired in rats with hippocampal lesions. Nature 297:681-683

Mumenthaler MS, Yesavage JA, Taylor JL, O'Hara R, Friedman L, Lee H, Kraemer HC (2003) Psychoactive drugs and pilot performance: a comparison of nicotine, donepezil, and alcohol effects. Neuropsychopharmacology. 28:1366-1373

Narahashi T, Moriguchi S, Zhao X, Marszalec W, Yeh JZ (2004) Mechanisms of action of cognitive enhancers on neuroreceptors. Biol Pharm Bull 27:1701-1706

Ogura H, Kosasa T, Kuriya Y, Yamanishi Y (2000) Donepezil, a centrally acting acetylcholinesterase inhibitor, alleviates learning deficits in hypocholinergic models in rats. Methods Find Exp Clin Pharmacol 22:89-95

Palmer AM (2002) Pharmacotherapy for Alzheimer's disease: progress and prospects. Trends Pharmacol Sci 23:426-433

Parent MB, Baxter MG (2004) Septohippocampal acetylcholine: involved in but not necessary for learning and memory? Learn Mem 11:9-20

Pepeu G, Giovannini MG (2004) Changes in acetylcholine extracellular levels during cognitive processes. Learn Mem 11:21-27

Petrosini L, Molinari M, Dell'Anna ME (1996) Cerebellar contribution to spatial event processing: Morris water maze and T-maze. Eur J Neurosci 8:1882-1896

Phillis JW (2005) Acetylcholine release from the central nervous system: a 50-year retrospective. Crit Rev Neurobiol 17:161-217

Poorheidari G, Stanhope KJ, Pratt JA (1998) Effects of the potassium channel blockers, apamin and 4-aminopyridine, on scopolamine- 
induced deficits in the delayed matching to position task in rats: a comparison with the cholinesterase inhibitor E2020. Psychopharmacology 135:242-255

Prickaerts J, Sik A, van der Staay FJ, de Vente J, Blokland A (2005) Dissociable effects of acetylcholinesterase inhibitors and phosphodiesterase type 5 inhibitors on object recognition memory: acquisition versus consolidation. Psychopharmacology 177:381-390

Rogers SL, Friedhoff LT (1996) The efficacy and safety of donepezil in patients with Alzheimer's disease: results of a US Multicentre, Randomized, Double-Blind, Placebo-Controlled Trial. The Donepezil Study Group. Dementia 7:293-303

Rogers SL, Doody RS, Mohs RC, Friedhoff LT (1998) Donepezil improves cognition and global function in Alzheimer disease: a 15-week, double-blind, placebo-controlled study. Donepezil Study Group. Arch Intern Med 158:1021-1031

Rose SP (2002) 'Smart drugs': do they work? Are they ethical? Will they be legal? Nat Rev Neurosci 3:975-979

Rupniak NM, Tye SJ, Field MJ (1997) Enhanced performance of spatial and visual recognition memory tasks by the selective acetylcholinesterase inhibitor E2020 in rhesus monkeys. Psychopharmacology 131:406-410

Sarter M, Givens B, Bruno JP (2001) The cognitive neuroscience of sustained attention: where top-down meets bottom-up. Brain Res Rev 35:146-160

Sarter M, Bruno JP, Givens B (2003) Attentional functions of cortical cholinergic inputs: what does it mean for learning and memory? Neurobiol Learn Mem 80:245-256

Saykin AJ, Wishart HA, Rabin LA, Flashman LA, McHugh TL, Mamourian AC, Santulli RB (2004) Cholinergic enhancement of frontal lobe activity in mild cognitive impairment. Brain 127:1574-1583

Seltzer B (2007) Donepezil: an update. Expert Opin Pharmacother 8:1011-1023

Shearman E, Rossi S, Szasz B, Juranyi Z, Fallon S, Pomara N, Sershen H, Lajtha A (2006) Changes in cerebral neurotransmitters and metabolites induced by acute donepezil and memantine administrations: a microdialysis study. Brain Res Bull 9:204-213

Shigeta M, Homma A (2001) Donepezil for Alzheimer's disease: pharmacodynamic, pharmacokinetic, and clinical profiles. CNS Drug Rev 7:353-368

Sonkusare S, Srinivasan K, Kaul C, Ramarao P (2005) Effect of donepezil and lercanidipine on memory impairment induced by intracerebroventricular streptozotocin in rats. Life Sci 77:1-14
Spowart-Manning L, van der Staay FJ (2004) The T-maze continuous alternation task for assessing the effects of putative cognition enhancers in the mouse. Behav Brain Res 151:37-46

Spowart-Manning L, van der Staay FJ (2005) Spatial discrimination deficits by excitotoxic lesions in the Morris water escape task. Behav Brain Res 156:269-276

Sugimoto H (2001) Donepezil hydrochloride: a treatment drug for Alzheimer's disease. Chem Rec 1:63-73

Sun QQ, Xu SS, Pan JL, Guo HM, Cao WQ (1999) Huperzine-A capsules enhance memory and learning performance in 34 pairs of matched adolescent students. Zhongguo Yao Li Xue Bao 20:601-603

Takada Y, Yonezawa A, Kume T, Katsuki H, Kaneko S, Sugimoto H, Akaike A (2003) Nicotinic acetylcholine receptor-mediated neuroprotection by donepezil against glutamate neurotoxicity in rat cortical neurons. J Pharmacol Exp Ther 306:772-777

Tokita K, Yamazaki S, Yamazaki M, Matsuoka N, Mutoh S (2002) Combination of a novel antidementia drug FK960 with donepezil synergistically improves memory deficits in rats. Pharmacol Biochem Behav 73:511-519

van der Staay FJ, Bouger PC (2005) Effects of the cholinesterase inhibitors donepezil and metrifonate on scopolamine-induced impairments in the spatial cone field orientation task in rats. Behav Brain Res 156:1-10

Winstein CJ, Bentzen KR, Boyd L, Schneider LS (2007) Does the cholinesterase inhibitor, donepezil, benefit both declarative and non-declarative processes in mild to moderate Alzheimer's disease? Curr Alzheimer Res 4:273-276

Wise LE, Iredale PA, Stokes RJ, Lichtman AH (2007) Combination of rimonabant and donepezil prolongs spatial memory duration. Neuropsychopharmacology 32:1805-1812

Yamada K, Takayanagi M, Kamei H, Nagai T, Dohniwa M, Kobayashi K, Yoshida S, Ohhara T, Takuma K, Nabeshima T (2005) Effects of memantine and donepezil on amyloid betainduced memory impairment in a delayed-matching to position task in rats. Behav Brain Res 162:191-199

Yesavage JA, Mumenthaler MS, Taylor JL, Friedman L, O'Hara R, Sheikh J, Tinklenberg J, Whitehouse PJ (2002) Donepezil and flight simulator performance: effects on retention of complex skills. Neurology 59:123-125

Zhang L, Zhou FM, Dani JA (2004) Cholinergic drugs for Alzheimer's disease enhance in vitro dopamine release. Mol Pharmacol 66:538-544 